\title{
Observation of a Metastable Cu Phase Formed at a Crystalline Si / Liquid Al-Alloy Interface in an Al-Cu-Mg-Si Alloy
}

\author{
James M. Howe ${ }^{1}$ and Matthew M. Schneider ${ }^{2}$ \\ 1. Department of Materials Science \& Engineering, University of Virginia, Charlottesville, VA 22904- \\ 4745, USA \\ 2. Advanced Materials Processing and Analysis Center, University of Central Florida, Orlando, FL \\ 32816, USA
}

I first became aware of Gareth Thomas when I accepted a position in the Metallography Section at Kaiser Aluminum and Chemical Company in Pleasanton, CA, in 1978. As a young research engineer, I was very interested in understanding precipitation processes in Al alloys, particularly the very early stages when precipitates are just nucleating and beginning to grow. As I researched various Al-alloy systems it quickly became apparent that Gareth had performed transmission electron microscopy (TEM) on just about all of the technologically important alloys, e.g., Al-Cu(-Mg), Al-Mg-Si, Al-Mg-Zn, as well as some model alloy systems of a more academic nature, e.g., Al-Ag. This included everything from the effects of solute-element additions on the formation of vacancy loops prior to precipitation, the nucleation mechanisms of precipitates on vacancy loops and dislocations, various sequences of metastable precipitate formation, the crystal structures of precipitates, their growth mechanisms and so forth (see [1] for example). All of this was exceptionally high-quality work, and looking back, it is easy to see how Gareth was applying the capabilities of TEM to understand basic mechanisms of material behavior to design better materials, a theme he followed his entire career.

While working at Kaiser Aluminum, I became convinced that if I was ever going to be able to understand precipitation processes in Al alloys, I needed to be able to see the atoms. So several years later I left Kaiser Aluminum to begin graduate studies at UC-Berkeley, investigating the atomic mechanisms of precipitate plate growth in Al-Ag alloy under the direction of Gareth and Ronald Gronsky, one of Gareth's former students and an expert in high-resolution TEM (HRTEM) at the National Center for Electron Microscopy. Of course, Gareth had already performed in-depth in-situ heating TEM studies of the mechanisms of precipitation in Al-Ag alloys [2], so needless to say, I had my work cut out. I completed my Ph.D., which focused on HRTEM investigation of Al-Ag precipitates, but Gareth's paper on in-situ TEM of Al-Ag stuck with me, so not long after graduation, I began to perform in-situ HRTEM heating experiments on Al alloys. I found that seeing processes happen in real time can be immensely revealing and this is a technique that I have continued to employ my entire career. This paper describes some recent results from in-situ heating HRTEM studies on the solid-liquid interface in an $\mathrm{Al}-\mathrm{Cu}-\mathrm{Mg}-\mathrm{Si}$ alloy [3].

Atomized particles of a hypereutectic Al-alloy (AA390) with Al-17.8Si-1.8Cu-0.6Mg (at.\%) were heated to $650{ }^{\circ} \mathrm{C}$ in-situ in a FEI Titan 80-300 TEM operating at $300 \mathrm{kV}$, using a Gatan Model 652.MA heating holder. Images were captured on a Gatan Multi-Scan 794 CCD camera mounted at the end of a Tridiem 863P Gatan Image Filter, using open-source CamStudio screen-capturing software installed on the TEM computer and synced with the CCD camera output and DigitalMicrograph ${ }^{\mathrm{TM}}$ software. Simulated HRTEM images were calculated using CrystalKit and MacTempas software (Total Resolution, LLC). 
Figure 1(a) is an experimental HRTEM image that shows the presence of $\{200\} \mathrm{Cu}$ planes at the interface between crystalline Si and liquid Al-alloy in the partially molten Al-Si-Cu-Mg sample. Small patches of such $\{200\} \mathrm{Cu}$ planes were observed to appear and disappear during video-recordings of the solid-Si/liquid Al-alloy interface at temperature. Figure 1(b) shows a simulated HRTEM image of the same interfacial phase and structure for comparison. The model for the simulation was constructed based on the following analysis. The crystalline $\mathrm{Si}$ is near a $<110>$ orientation and one set of $\{111\}$ planes is highly visible in the image. The Si has a $\{113\}$ solid-liquid interface plane, and the orientation between the crystalline $\mathrm{Si}$ and $\mathrm{Cu}$ is such that the $\{200\} \mathrm{Cu}$ planes are rotated 148 degrees counterclockwise from the $\{111\}$ Si planes. Given this information, the stereographic projection of $\mathrm{Cu}$ was used to search for potential matching interface planes with the $\{113\}$ plane of Si. Possible candidates for the matching plane in $\mathrm{Cu}$ were then evaluated in terms of their degree of atom-row matching. Figure 2 shows a $\{076\} \mathrm{Cu}$ plane superimposed onto a $\{113\} \mathrm{Si}$ interface plane, with the atom positions represented in blue and orange circles, respectively. With the close-packed directions in each phase aligned vertically, 12 of the $27 \mathrm{Si}$ rows matches $\mathrm{Cu}$ atom rows to within a $15 \%$ spatial tolerance, for a $44 \%$ match. This interfacial configuration was determined to be the best match of all possibilities explored. Thus, the orientation relationship between the metastable $\mathrm{Cu}$ phase at the solid Si/liquid Alalloy interface was determined as $\{113\}_{\mathrm{Si}}||\{076\}_{\mathrm{Cu}} ;\left\langle 110>_{\mathrm{Si}}||<100>_{\mathrm{Cu}}\right.$. These results are consistent with previous analytical TEM measurements, which showed evidence of $\mathrm{Cu}$ segregation to the interface [4], but they also show that the $\mathrm{Cu}$ is not segregated uniformly across the interface, and indicate that the metastable $\mathrm{Cu}$ patches may serve as a precursor phase to nucleation of equilibrium $\mathrm{Cu}-$ rich phases. These and other aspects of the $\mathrm{Cu}$ patches, particularly regarding their dynamic behavior, will be described in this presentation [5].

\section{References:}

[1] A. Kelly and R. B. Nicholson, "Precipitation Hardening," Pergamon Press, Oxford (1963) p. 178.

[2] J. A. Hren and G. Thomas, Trans. Met. Soc. AIME 227 (1963) p. 308.

[3] M. M. Schneider, M.S. Thesis, University of Virginia, December (2014).

[4] P. Palanisamy and J. M. Howe, Acta Mater. 61 (2013) p. 4339.

[5] It is a great pleasure to acknowledge and thank Gareth Thomas for his many contributions, as well as support of this research by the National Science Foundation under Grant DMR-1106230.

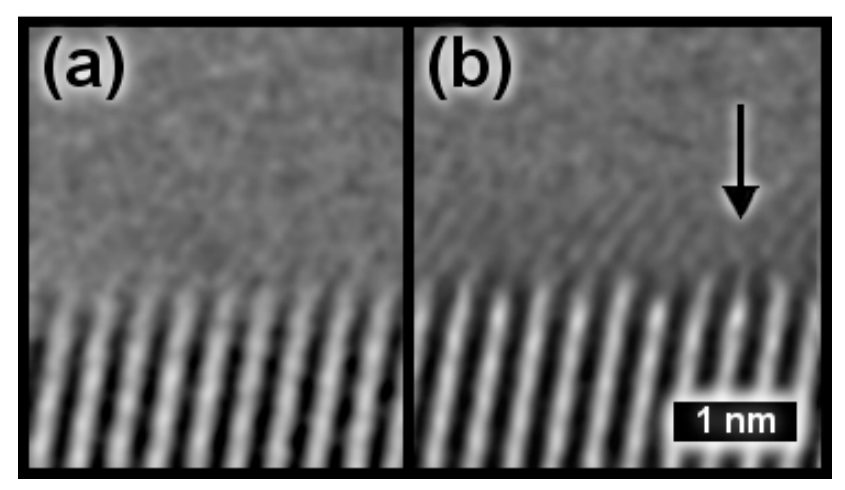

Figure 1. (a) Comparison between an experimentally observed HRTEM image, and (b) a simulated image, of $\{076\} \mathrm{Cu}$ on the $\{113\} \mathrm{Si}$ solid/liquid interface [3].

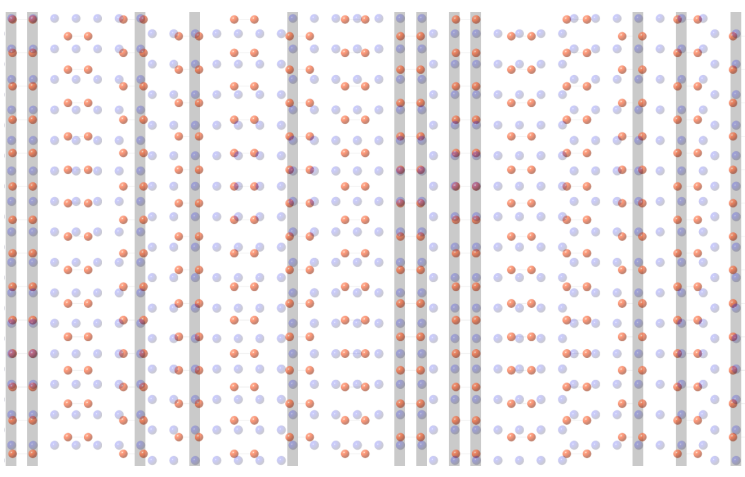

Figure 2. Schematic showing the atom-row matching between $\{113\} \mathrm{Si}$ atom positions (orange) and $\{076\} \mathrm{Cu}$ atom positions (blue). Vertical rows matching within $\pm 15 \%$ are highlighted [3]. 